\title{
Research Groups: How big should they be?
}

Isabelle Cook, Sam Grange, Adam Eyre-Walker

Understanding the relationship between scientific productivity and research group size is important for deciding how science should be funded. We have investigated the relationship between these variables in the life sciences in the United Kingdom using data from 398 principle investigators (PIs). We show that three measures of productivity, the number of publications, the impact factor of the journals in which papers are published and the number of citations, are all positively correlated to group size, although they all show a pattern of diminishing returns - doubling group size leads to less than a doubling in productivity. The relationships for the impact factor and the number of citations are extremely weak. Our analyses suggest that an increase in productivity will be achieved by funding more Pls with small research groups, unless the cost of employing post-docs and PhD students is less than $20 \%$ the cost of a PI. We also provide evidence that post-docs are more productive than PhD students both in terms of the number of papers they produce and where those papers are published. 
Research Groups: How big should they be?

3

8

*Corresponding author: a.c.eyre-walker@sussex.ac.uk 
Introduction

20 How large should a research group be? Should resources be concentrated into a small

21 number of research groups or should funding be more evenly distributed? This question has

22 been investigated in a number of different countries at a variety of different levels of

23 organisation. Most analyses of individual research groups, rather than departments or

24 universities, have found that the number of research papers per group member, is either

25 unrelated to (Cohen 1981; Johnston et al. 1995; Seglen \& Aksnes 2000) or that it declines

26 with group size (Brandt \& Schubert 2013; Carayol \& Matt 2004; Diaz-Frances et al. 1995).

27 Reports that there is an optimal group size (Qurashi 1984; Qurashi 1993; Stankiewicz 1979)

28 appear to have limited statistical support (see for example Cohen's (Cohen 1984) criticism of

29 Qurashi (Qurashi 1984)), as do reports that productivity increases exponentially with research

30 group size (Wallmark et al. 1973) (see criticism by (Cohen 1981)).

32 The question of research group size and the allied question of funding has been brought back

33 into focus with a recent analysis of National Institute of Health (NIH) data. Jeremy Berg, a

34 former director at the NIH, found that both the number of papers and the median impact factor

35 (IF) of papers increased with $\mathrm{NIH}$ funding per lab until a maximum was attained at

36 approximately $\$ 750,000$ per year, after which both the number of publications and the median

37 IF declined (see https://loop.nigms.nih.gov/2010/09/measuring-the-scientific-output-and-

38 impact-of-nigms-grants/, reported by (Wadman 2010)). This has led to a policy by which

39 grants from well-funded labs are subject to additional review by the NIH (Berg 2012).

40 However, Berg presented no statistical evidence in support of the maximum. A truer reflection

41 of the data might be that groups with low levels of funding are relatively productive and that

42 funding explains little of the variance in publication rate or where those papers get published.

43 A recent analysis of the Canadian funding of science has come to a very similar conclusion

44 (Fortin \& Currie 2013). Various measures of productivity and impact, including the number of

45 publications and the number of citations, are positively correlated to the level of funding, but

46 the relationship is very weak, both in terms of the slope of the relationship and the variance

47 explained. Furthermore, the relationship is one of diminishing returns; productivity increases

48 with funding but not proportionally (Fortin \& Currie 2013). 
50 Here we consider the current relationship between research group size and productivity in the

51 Biological Sciences in the United Kingdom. We consider several measures of productivity: the

52 number of papers published by a research group, the impact factor of the journals in which

53 those papers are published and the number of citations they receive as a function of group 54 size.

\section{Materials and Methods}

58 Group size

59 We emailed all principal investigators (PIs) in biological science departments in universities 60 that had made a return to sub-panel 14 (Biological Sciences) of the 2008 edition of the 61 Research Assessment Exercise. Email addresses were harvested from departmental web62 sites. Emails were sent in two phases, in October 2012 and in October 2013 by IC and SG 63 respectively. Contactees were asked to provide the number of post-docs, PhD students, 64 technicians and other staff working in their group. If individuals were part-time or shared 65 between faculty we asked that they be counted as a fraction of a full time equivalent.

66 Contactees were also asked whether they had been at the same institution over the 67 preceeding 5 years, and only individuals that fullfilled this criterion were included in 68 subsequent analysis; this was to make it possible to identify the publications produced by 69 each PI. Copies of the emails sent to PIs are included as supplementary information.

\section{Publication data}

72 The publications published by a PI were obtained by searching the ISI Web of Science

73 database by employing their author search facility using last name, first initial and institutional

74 address, restricting the search to papers published in the life sciences. To check the data, we

75 listed the initials or first names associated with each paper returned by the initial search - for

76 example a search of Jones, $\mathrm{C}$ at Dundee might return publications from Jones, Chris and 77 Jones, Cate at Dundee. Publication files containing multiple authors were manually curated.

78 We also checked all publication files that had papers with more than 20 authors since the 79 address field in the initial search is not directly associated with the author - for example, a 80 search of Jones, $C$ at Dundee might return a paper by Jones, $C$ at Cambridge, who co- 
81 authored a paper with someone at Dundee. Such mistakes are more likely for papers with

82 many authors. The publication data was downloaded by AEW in July and August 2014,

83 however only papers published between and including 2008 to 2012 were considered for

84 scientists contacted in 2012, and between and including 2009 to 2013 for scientists contacted

85 in 2013. For each publication we divided the number of citations by the number of years since

86 publication (e.g. the citations published for a paper in 2012 for individuals censused in 2012

87 would be divided by one, those from 2011 by 2 ...etc). We also counted the number of authors

88 for each paper and obtained the impact factor of the journal in was published using impact

89 factors from 2013.

91 Statistics

92 Because all the variables in this analysis have skewed distributions, we log transformed the

93 data before performing normal least squares regression. However, we also performed

94 regressions on untransformed data to gain further insight into the relationship between the

95 number of papers and group size. For this we assumed the dependent variable was Poisson

96 distributed and we estimated the parameters of the regression model by maximum likelihood;

97 note that this differs from standard Poisson regression because we are assuming that the

98 untransformed dependent variable is a linear function of the dependent variables, whereas

99 regular Poisson regression assumes the log of the dependent variable is a linear function.

100 Differences between nested models were tested using likelihood ratio tests and differences

101 between un-nested models were assessed using the Akaike Information Criterion (AIC).

102

103 To test whether the regression coefficients (i.e. the slopes associated with PhDs, post-docs, 104 technicians and other scientists) were significantly different to each other in a multiple 105 regression we bootstrapped the data by $\mathrm{PI}$, re-running the multiple regression calculating the 106 difference between the regression coefficients each time. We repeated this 1000 times. The 107 p-value was the proportion of differences between regression coefficients that were greater or 108 less than zero, as appropriate (e.g. in testing whether the number of papers is more strongly 109 dependent upon the number of post-docs and PhD students, where the regression coefficient 110 for post-docs is greater than that for PhD students, the p-value was the number of differences 111 between the bootstrap coefficients that were negative). 
113 Data availability

114 The anonymised dataset is available in supplementary table S1.

116 Ethical considerations

117 It was not considered necessary to submit this study for ethical review given the nature of the

118 project - simply requesting research group size information directly from Pls. All participants

119 gave their written consent in the form of an email reply. All data was treated as confidential.

\section{Results}

122 In order to investigate the relationship between scientific productivity and research group size 123 within the biological sciences in the United Kingdom we contacted all principal investigators $124(\mathrm{Pl})$ who were in UK research departments that took part in the 2008 Research Assessment 125 Exercise. In total 2849 academics were contacted personally by email, of which $398(10 \%)$ 126 replied and had been at the same institution over the previous 5 years. We required them to 127 have been present at the same institution so that we could obtain their publication record over 128 that period (see Materials and Methods). We asked them how many PhD students, post-docs, 129 technicians and other researchers (mostly research associates) they had in their research 130 group on the day they were contacted (hereafter we define a group as the principal 131 investigator (PI) and their associated post-docs, PhD students, technicians and other 132 research staff (usually pre-doctoral research assistants)). We subsequently downloaded their 133 publications from the previous 5 years from the Web of Science along with the number of 134 citations each paper had received over a period of 6 or 7 years, depending on when the PI 135 was contacted (downloading of the Web of Science data was done sometime after the Pls 136 were contacted). The number of citations for each paper was divided by the number of years 137 since publication.

139 Group size

140 Most biology research groups in the UK are of modest size, containing less than 10 staff and 141 students, including the PI (Figure 1). The mean research group size is 7.3 (standard deviation 142 of 4.5 ) with a range of 1 to 31 . On average a research group contains $3.0 \mathrm{PhD}$ students, 2.1 
143 postdocs, 0.5 techinicians and 0.68 other staff (mostly research associates). The numbers of

144 post-docs, $\mathrm{PhD}$ students, technicians and other staff are mildly but significantly positively

145 correlated to each other, with the exception of PhD students and other staff (Table 1).

147 Models

148 Since most of the variables in our analysis have strongly skewed distributions we log

149 transformed the data, adding one where the variable contained zeros (this only applied to the 150 number of publications, and the numbers of PhD students, post-docs, technicians and other 151 researchers). This yielded approximately normally distributed variables. As a consequence, in 152 fitting a linear model to the log transformed data we are fitting a model of the following form:

$154 y=a x^{b}$

156 since

$\log (y)=\log (a)+b \log (x)$

The slope of the relationship between $\log (y)$ and $\log (x)$ indicates whether $y$ increases proportionally with $\times(b=1)$, less than proportionally with $x(b<1)$ (i.e. diminishing returns, or decreasing returns to scale), or more than proportionally with $x(b>1)$ (i.e. increasing returns or increasing returns to scale). Unless otherwise clearly stated, references to a variable will mean the log of that variable - e.g. the number of papers is correlated to group size means the log of the number of papers is correlated to the log of group size.

168 The average number of publications published by each group in the previous five years was 16922.0 papers ( $S D=18.8$ ) but varies considerably between Pls, from 0 to 177 . The number of 170 publications over the preceding 5 years is significantly correlated to the total group size $(r=$ $1710.43, p<0.001$ )(Figure 2). However, group size explains less than $20 \%$ of the variance in the 172 number of papers, and at all levels of group size there is substantial variance in the number of 173 papers produced (Figure 2). The slope of the relationship between the number of papers and 
174 group size is significantly less than one $(b=0.57$ (SE $=0.06), p<0.001)$ indicating a 175 diminishing returns relationship; i.e. the number of papers increases with group size but less 176 than proportionally. This is not simply due to adding one to the number of papers before log 177 transforming, because if we do not add one and drop the one group with no research papers, 178 we get qualitatively the same relationship: $b=0.62, p<0.001)$. The diminishing returns 179 relationship can be illustrated simply by dividing the (untransformed) number of papers by the 180 (untransformed) group size; the number of papers per group member decreases as group 181 size increases (Spearman's rank correlation $=-0.20, p<0.001$ )(Figure 3).

183 A multiple regression suggests that the number of published papers is significantly and 184 positively correlated to the number of $\mathrm{PhDs}(\mathrm{p}<0.001)$, post-docs $(p<0.001)$ and other researchers $(p=0.001)$, but not the number of technicians $(p=0.47)$. The slope associated with post-docs $(b=0.39)$ is considerably larger than the slope associated with either PhD students $(b=0.24)$ or other researchers $(b=0.22)$. In the case of post-docs versus other members this difference is significant $(p=0.032)$ and almost significant for PhDs versus post-docs $(p=$ 0.058). The slopes suggest that post-docs are on average more productive than $\mathrm{PhD}$

In the biological sciences most papers are co-authored, often with a large number of coauthors - the mean number of authors per paper considered here is 9.3. As a consequence the number of papers may not reflect the output of a particular research group but the collaborations the group participates in. We therefore also considered the number of papers in which the PI was first or last author (these are traditionally the places where the lead PI on 197 a project will appear in the biological sciences). On average each PI produced 11.6 (SD = 10.0) first and last author papers, which means that about half of all papers associated with a $\mathrm{PI}$ are first and last author papers. However, the proportion of papers that are first author papers varies significantly between Pls (Chi-square $=1455, \mathrm{df}=378, \mathrm{p}<0.001)$. The paper $(r=-0.18, p<0.001)$, but it is not correlated to group size $(r=-0.019, p=0.70)$. 
204 The number of first and last author papers is significantly correlated to group size $(r=0.39$, $205 p<0.001)$, with a slope $(0.56, p<0.001)$ which is similar to that observed for the total number of 206 publications, indicating diminishing returns with increasing group size. The number of first and 207 last author papers is significantly corelated to the number of $\mathrm{PhD}$ students $(b=0.33, p<0.001)$ 208 and post-docs $(b=0.41, p<0.001)$, but not other members and the number of technicians.

209 The slopes for PhDs and post-docs are not significantly different to each other in this analysis $210 \quad(p=0.24)$.

212 Impact versus group size

213 Large resrach groups produce more papers than small groups, but do they also produce 214 papers that appear in journals with higher IFs and which gain more citations? We find that the 215 mean IF of the papers associated with a PI is significantly correlated to group size $(r=0.14$, $216 p=0.004$ )(Figure 4). However, the correlation is very weak - group size only explains $2 \%$ of 217 the variance - and the slope is very shallow $(b=0.10, p=0.004))$. Surprisingly we find that 218 the mean IF is positively correlated to the number of post-docs $(b=0.22, p<0.001)$ and other 219 researchers $(b=0.087, p=0.022)$, but is negatively correlated to the number of PhD students $220(b=-0.16, p<0.001)$. These slopes are significantly different to each other (PhDs versus 221 post-docs, $p<0.001$, PhDs versus others, $p<0.001$, post-docs versus others $p=0.02$ ). 222

223 Much the same pattern holds for the number of citations per year. The mean log number of 224 citations per year is significantly correlated to group size $(r=0.15, p=0.004)$ (Figure 5).

225 However, the regression explains very little of the variance and the slope is very shallow $(b=$ $2260.12(0.04))$. The number of citations is only significantly correlated to the number of post$227 \operatorname{docs}(b=0.19, p<0.001)$. 


\section{Discussion}

232 We have shown that the number of papers published by a group, and the mean IF and the

233 mean number of citations of those papers, are all positively correlated to group size. However,

234 the slopes are very shallow for the IF and the number of citations, indicating that group size

235 has little effect on where papers are published and how many citations they receive. For all

236 three variables the slope of the relationship between the log of the variable and the log of

237 group size is less than one, indicating that each variable increases with group size, but less

238 than proportionately; i.e. there is diminishing returns such that doubling the group size leads

239 to less than a doubling in measures of productivity.

241 A diminishing returns relationship could potentially be due to two non-mutually exclusive

242 factors. It could be that all group members are equally productive, but that increasing group

243 size leads to inefficiencies that lead to a reduction in the productivity per individual.

244 Alternatively it might be that the PI has a larger effect on productivity than other group

245 members. Since there is generally only one PI per group, increasing group size leads to a

246 dilution of this contribution and hence diminishing returns. Given that post-docs appear to be

247 significantly more productive than $\mathrm{PhD}$ students, it seems quite possible that Pls contribute

248 more to productivity than post-docs, given their years of additional training, and that

249 diminishing returns arises because of this.

251 To investigate the reasons for the diminishing returns relationship we fit a series of models to

252 the untransformed data using maximum likelihood assuming the error term was Poisson

253 distributed (this is different to Poisson regression in which the log of the dependent variable is

254 assumed to be a linear function of the independent variable). We concentrated on the

255 relatiomship between the number of papers and group size because the other variables are

256 only very weakly correlated to group size. We subtracted the PI from the group size because

257 groups cannot have zero members. First, we compared a simple linear model $(y=a+b x)$ to

258 a model including a quadratic term $\left(y=a+b 1 x+b 2 x^{2}\right)$; the inclusion of the quadratic term

259 did not significantly improve the fit of the model (log likelihood for the linear model $=-2927.89$,

260 for quadratic model $=-2927.35$ ) suggesting that the number of publications increases linearly

261 with group size. However, one might argue that the quadratic model simply fails to improve 
262 the fit because it is an inadequate model. We therefore fit a very different model of the form a

$263+b \mathrm{e}^{-c x}$; this equation allows $y$ to be linearly related to $x$ (when $c$ is small) or to show

264 diminishing or increasing returns, all with with arbitrary intercepts. This model fits the data

265 slightly, but not significantly, better than the simple linear model (log likelihood $=-2927.47$ ).

266 However, the value of $c$ is very small so this model is essentially linear; the estimated

267 relationship is $313.5-303.7 \mathrm{e}^{-0.0065 x}$ which is approximately $313.5-303.7(1-0.0065 x)=$

$2689.8+2.0 x$. This is very similar to the simple linear model, $y=10.1+1.9 x$, fitted above.

269 These analyses therefore suggest that the diminishing returns does not arise because larger

270 groups become inefficient, because the evidence suggests that each additional member

271 increases productivity by the same amount as other members. Instead diminishing returns

272 seems to arise because the PI contributes significantly more to productivity than other team

273 members, at least when group size is small. We cannot say anything about the contribution of

274 the PI in larger groups because the contribution of the PI to productivity might be a function of

275 group size; for example, the contribution of the PI might decline as group size increases.

277 What do the results imply for the funding of science? Although, we have found that all

278 measures of productivity increase with group size, they do not do so proportionally; there is

279 diminishing returns. This would seem to suggest that we should favour small groups.

280 However, it may be that some types of science can only be conducted by large research

281 groups. Furthermore, Pls are more expensive to employ than other team members, so it

282 might pay to employ more post-docs and PhD students than Pls. For example, if the average

283 cost of employing other group members is $20 \%$ that of a PI then there no evidence of a

284 diminishing returns relationship between the $(\log )$ of the number of papers and $(\log )$ cost

285 since the slope $=1.0$. If the relative cost is $10 \%$ there is a pattern of increasing returns

286 because the slope $=1.5$. Although, we do not know the average cost of employing Pls, post-

287 docs and PhD students it seems unlikely that Pls cost more than 5-times as much as the

288 average post-doc and $\mathrm{PhD}$ student, at least not in the UK. The data therefore suggest that the

289 best policy in terms of scientific productivity is to invest in more PIs, rather than post-docs and

290 PhD students. Such a policy would also go someway to addressing the poor career prospects

291 amongst PhD students and post-docs within academia. In the UK only $3.5 \%$ of science PhDs

292 gain a permanent academic position and $17 \%$ go into non-university research, with about 
$29379.5 \%$ ending up outside scientific research completely (The Royal Society 2010). However, it

294 should be appreciated that recruiting more Pls is likely to reduce the average quality of PIs.

296 Our results are consistent with several previous studies in which productivity has shown a 297 diminishing returns relationship with research group size for scientists in Germany, France 298 and Mexico (Brandt \& Schubert 2013; Carayol \& Matt 2004; Diaz-Frances et al. 1995); a 299 similar pattern is also evident between measures of productivity and research grant income 300 (Fortin \& Currie 2013; Wadman 2010) (note that Berg did not explicitly test for diminishing 301 returns in his analysis of $\mathrm{NIH}$ data, but the linear regression had a significantly positive 302 intercept which would yield a diminishing returns relationship on a log scale). In contrast, 303 Cohen (Cohen 1981) and Seglen and Aksnes (Seglen \& Aksnes 2000) reported a linear 304 relationship between the untransformed number of papers and untransformed research group 305 size with an intercept that was close to or not significantly different to zero. In the case of the 306 analyses performed by Cohen this may have been due to power because for each of his 307 three datasets he had rather little data.

309 We have also presented evidence that post-docs are more productive than PhD students. 310 They produce more papers and those papers are published in journals with (slightly) higher

311 IFs; in fact the mean IF significantly decreases as the number of $\mathrm{PhD}$ students increases, 312 although the decrease is very slight. The number of citations is also only significantly 313 correlated to the number of post-docs but not PhDs. To obtain a more quantitative estimate of 314 how much more productive post-docs were than PhD students in terms of the number of 315 papers we re-ran the multiple regression on the untransformed data using the Poisson 316 regression model. The slopes suggest that each post-doc adds 3.48 papers per 5 years 317 whereas PhD students and other researchers add 1.53 and 1.98 papers respectively. The 318 slopes are significantly different between PhDs and post-docs in the untransformed data $(p=$ 319 0.004), but not between other researchers and either post-docs or PhDs. It is perhaps not 320 surprising that post-docs are more productive than $\mathrm{PhD}$ students since post-docs have more 321 training and there is likely to be some degree of selectivity in which PhD students become 322 post-docs. 
324 Our definition of produtivity is limited. In particular we do not take into account the role of PIs

325 as teachers and the conribution that post-docs and $\mathrm{PhD}$ students make in careers other than

326 academic research. Furthermore we do not measure other forms of productivity such as

327 patents and policy documents. Our results must be interpreted in the light of these caveats.

328

329 Although we have collected data from a large number of groups, we have relied upon self330 reporting. This might have potentially biased the results. In particular we may have had 331 under-reporting from small groups or groups that were unproductive. It is difficult to address 332 this problem. Site visits to selected universities may help, but even then there is no guarantee 333 of complete or unbiased results. We have also restricted our analysis to Pls that have 334 remained at the same instutition for 5 years; this might have biased our results away from 335 young researchers, who may move early in their career.

337 In summary we have shown that three measures of productivity, the number of papers, the 338 impact factor and the number of citations, increase with group size. However, they all show a pattern of diminishing returns and the relationships are weak in terms of the variance that group size explains; this is particularly the case for the impact factor and the number of citations. Our analyses support a funding model in which productivity is maximised by having many small groups rather concentrating resources into a few large ones.

Acknowledgements: we are grateful to Torben Schubert, Jon Lorsch and Michael Lauer for helpful discussion and two referees, David Currie and Christian Althaus, for useful comments on the manuscript. We are also very grateful to all those academics who responded to our

\section{References}

351 Berg JM. 2012. Well-funded investigators should receive extra scrutiny. Nature 489:203-203. 352 Brandt T, and Schubert T. 2013. Is the university model an organizational necessity? Scale and agglomeration effects in science. Scientometrics 94:541-565. 
354 Carayol N, and Matt M. 2004. Does research organization influence academic production? Laboratory level evidence from a large European university. Research Policy 33:10811102.

Cohen JE. 1981. Publication Rate as a Function of Laboratory Size in 3 Biomedical-Research Institutions. Scientometrics 3:467-487.

Cohen JE. 1984. Statistical-Theory Aids Inference in Scientometrics - (Comments to Publication Rate as a Function of the Laboratory Group-Size by Qurashi,Mm.). Scientometrics 6:27-32.

Diaz-Frances E, Ruiz-Velasco S, and Jimenez J. 1995. Relationship between publication rate and research group size in Mexico. Fifth Biennial Conference of the International Society for Scientometrics and Informetrics: Learned Information. p 137-146.

Fortin JM, and Currie DJ. 2013. Big Science vs. Little Science: How Scientific Impact Scales with Funding. PLOS ONE 8.

Johnston R, Grigg L, and Currie J. 1995. Size versus performance in research. Australian Universities Review 2:60-64.

Qurashi MM. 1984. Publication Rate as a Function of the Laboratory Group-Size. Scientometrics 6:19-26.

Qurashi MM. 1993. Dependence of Publication-Rate on Size of Some University Groups and Departments in Uk and Greece in Comparison with Nci, USA. Scientometrics 27:19-38. Seglen PO, and Aksnes DW. 2000. Scientific productivity and group size: A bibliometric analysis of Norwegian microbiological research. Scientometrics 49:125-143.

Stankiewicz R. 1979. The size and age of Swedish academic research groups and their scientific performance. In: Andrews FM, ed. Scientific productivity: the effectiveness of research groups in six countries. Cambridge: VCambridge University Press.

The_Royal_Society. 2010. The scientific century: securing our future prosperity. London: The Royal Society.

Wadman M. 2010. Study says middle sized labs do best. Nature 468:356-357.

Wallmark JT, Eckerste.S, Langered B, and Holmqvis. He. 1973. Increase in Efficiency with 383 
Table $\mathbf{1}$ (on next page)

Correlations between group members

Table 1 - The correlations between the numbers of PhD students, post-docs, technicians and other group members, and the levels of significance from test of whether the correlation is significantly different from zero $(* p<0.05, * * * p<0.001)$ 


\begin{tabular}{|l|l|l|l|}
\hline & Post-doc & Technician & Other \\
\hline PhD & $0.27^{* * *}$ & $0.18^{* * *}$ & 0.017 \\
\hline Post-doc & & $0.21^{* * *}$ & $0.12^{*}$ \\
\hline Technician & & & $0.24^{\star * *}$ \\
\hline
\end{tabular}

2 
1

The distribution of group size

Figure 1 - The distribution of group size amongst 398 Pls within the Life Sciences in the United Kingdom.

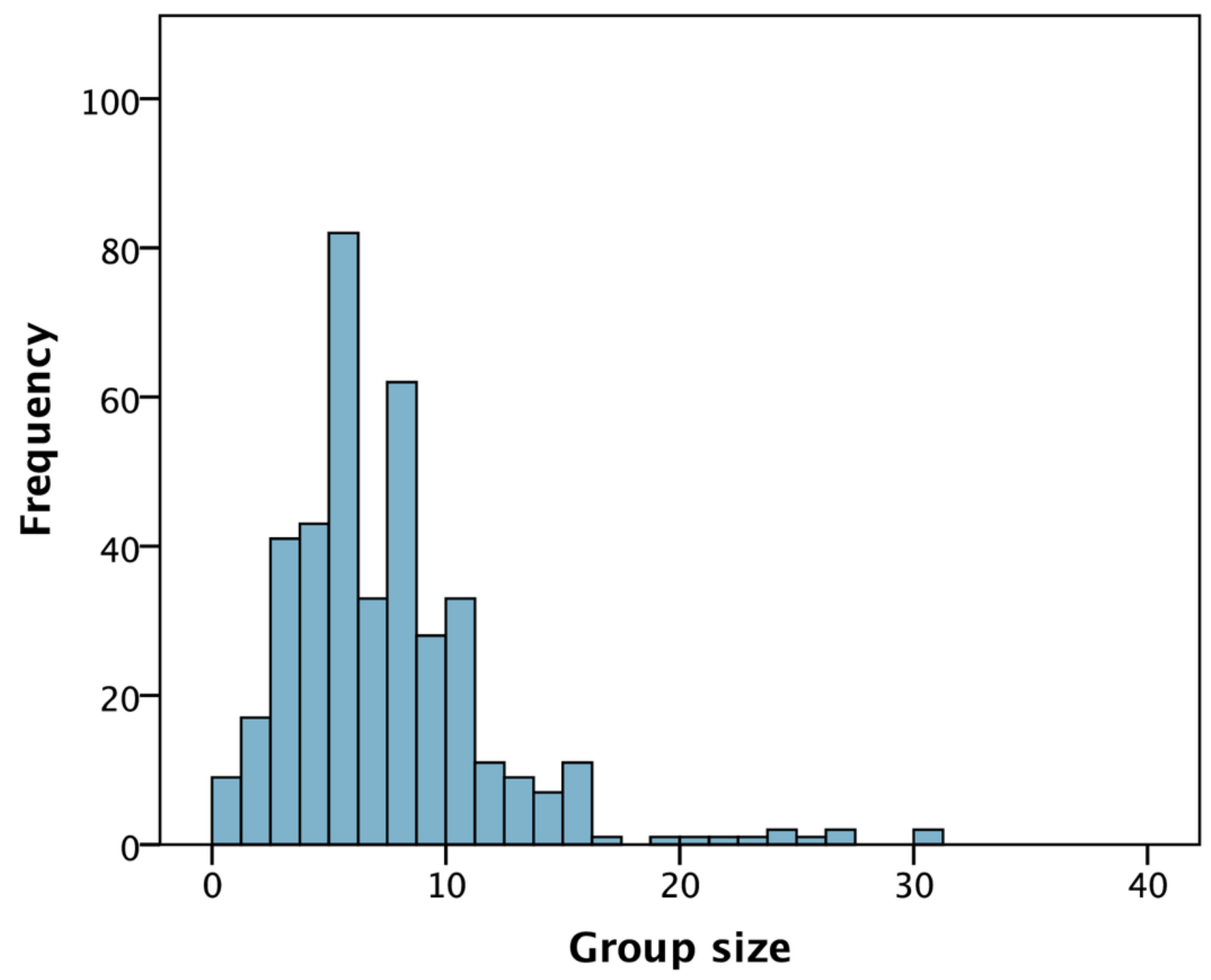


2

Number of papers versus group size

Figure $\mathbf{2}$ - The number of papers versus group size. The least squares line of best fit is shown.

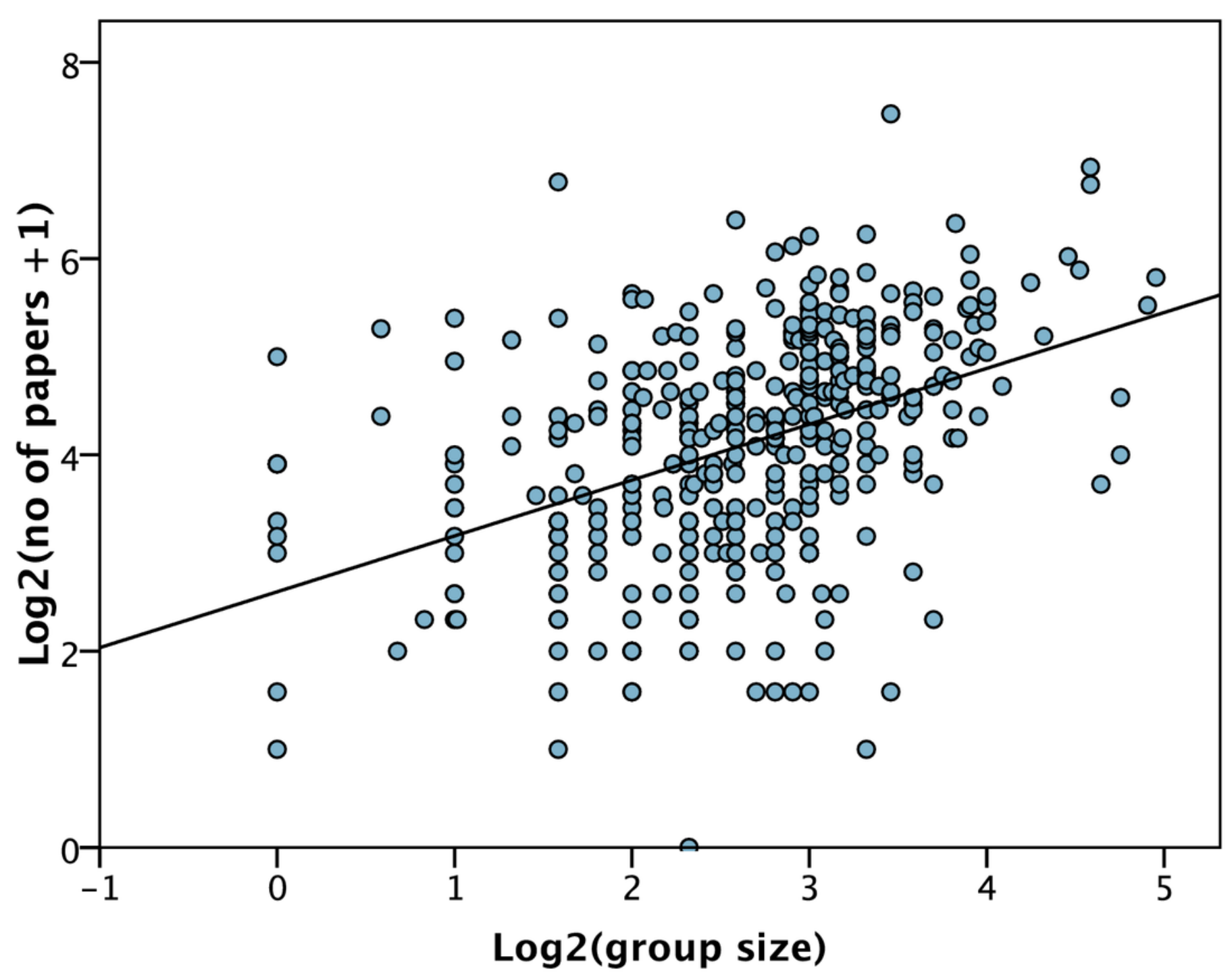


3

Paers per group member versus group size

Figure 3 - The number of publications per group member versus group size.

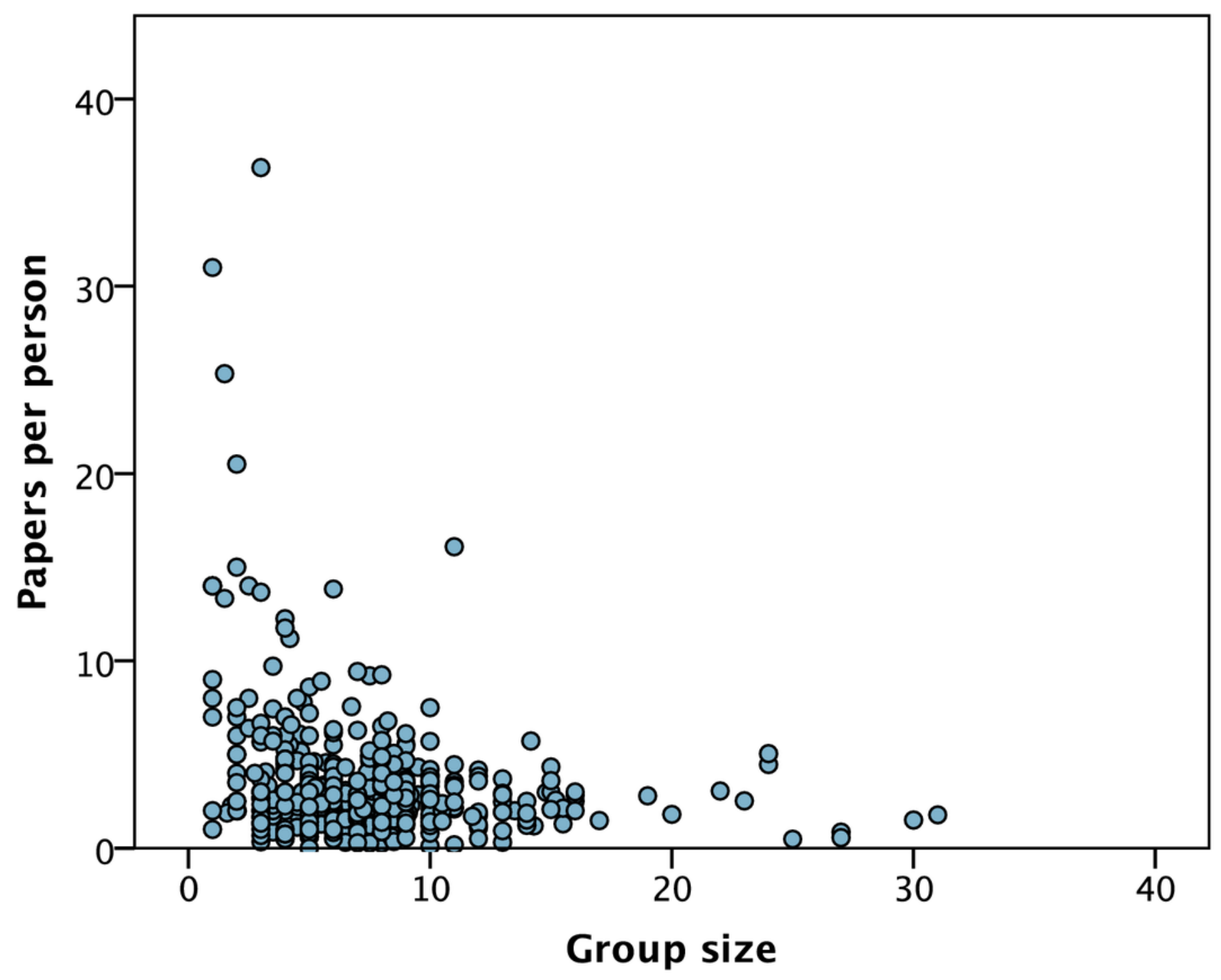


4

IF versus group size

Figure 4 - IF versus group size. The least squares line of best fit is shown.

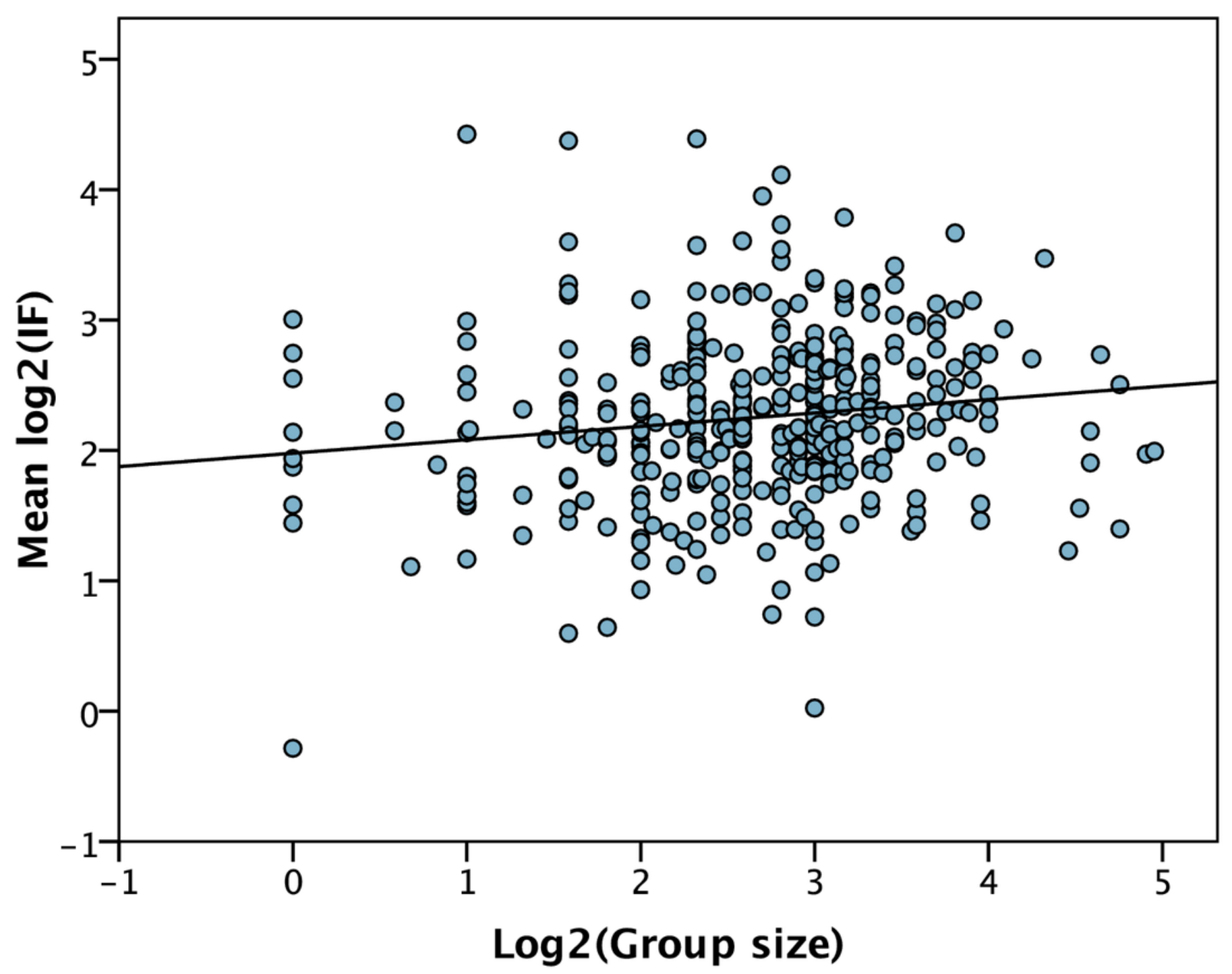




\section{5}

Number of citations versus group size

Figure $\mathbf{5}$ - Number of citations per year versus group size. The least squares line of best fit is shown.

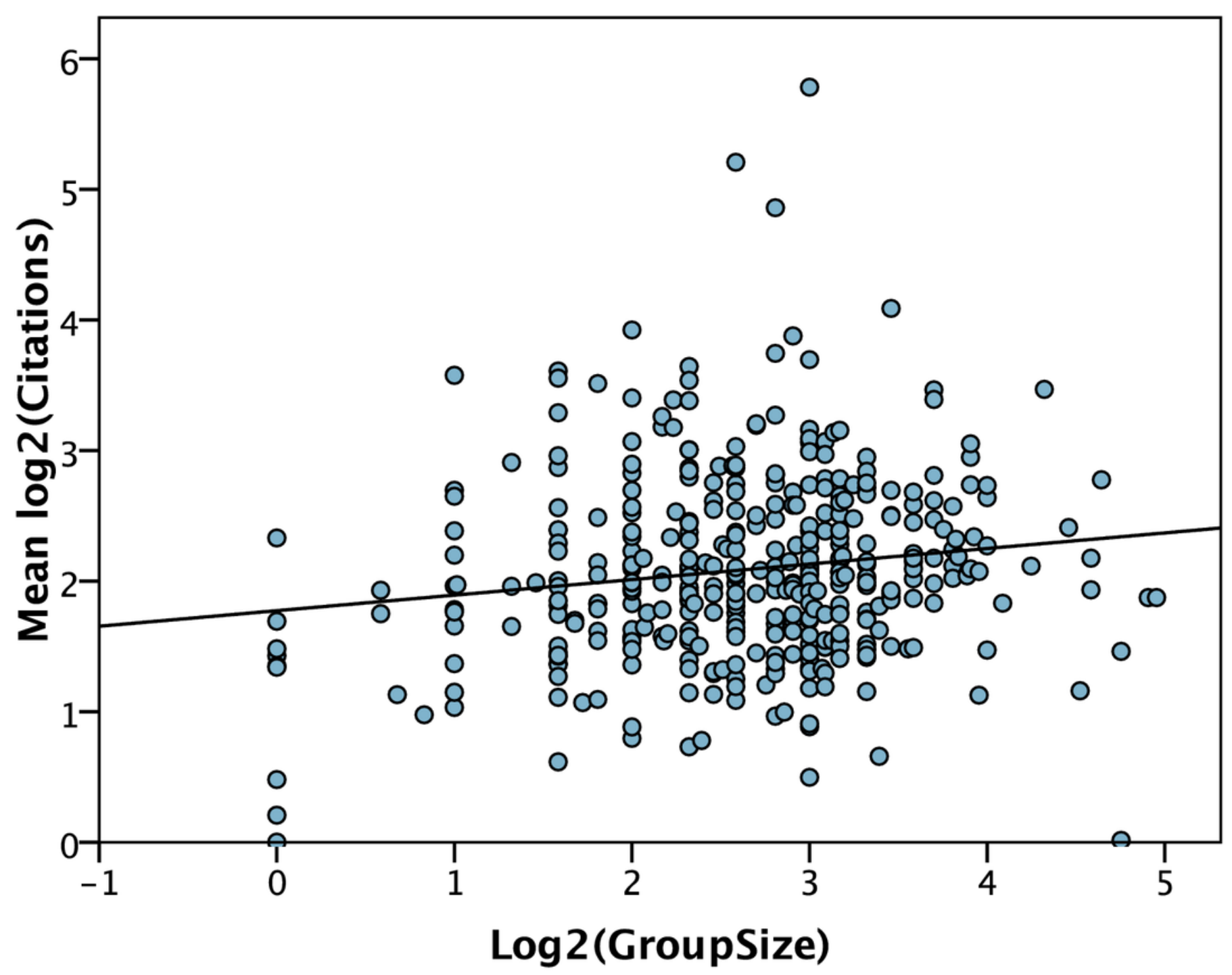

